\section{Le lapin. De la biologie à l'élevage}

\section{Gidenne T. (coord.), Éditions Quæ, Collection « Savoir faire ", 2015, 270 p., ISBN 9782759224166}

Voici un ouvrage fort singulier dédié au lapin, petite espèce animale, grande d'intérêt pour l'élevage et la nutrition humaine. L'apport indéniable de cet écrit réside tout particulièrement dans l'échange continu qu'entretiennent les auteurs entre les connaissances scientifiques les plus récentes obtenues sur les différentes fonctions biologiques de cette espèce et leur exploitation pratique dans son élevage intensif. À cet égard, l'ouvrage s'articule fort logiquement sur une structure où chacune des fonctions de base de l'animal est présentée. Sept chapitres se succèdent : 1) anatomie, taxonomie, origine, évolution et domestication, 2) physiologie, 3) reproduction, 4) habitat et comportement, 5) nutrition et alimentation, 6) santé et prévention des maladies et 7) génétique et sélection. Chaque chapitre est rédigé par une équipe d'auteurs ayant consacré de nombreuses recherches à ces fonctions biologiques. Par conséquent, les chapitres s'appuient sur une bibliographie conséquente basée en majorité sur des références très récentes, en majorité publiées dans des revues scientifiques reconnues à l'échelle mondiale, et que le lecteur le plus curieux pourra consulter pour un approfondissement de ses connaissances.

L'intérêt de l'ouvrage réside tout particulièrement dans l'exposé des avancées les plus récentes de la cuniculture intensive, issues justement de l'accumulation de connaissances scientifiques dans ce domaine. Ainsi, le lecteur pourra retrouver les derniers développements adoptés par les éleveurs de lapins en matière de rythme de reproduction et les usages répandus de l'insémination artificielle chez cette espèce. De même, l'exploitation du comportement social des lapins et de ses conséquences sur son logement en élevage intensif est rapportée. Par ailleurs, un long récapitulatif des connaissances les plus récentes sur l'alimentation du lapin, au cours des différents stades physiologiques de sa carrière, est présenté. Ainsi, l'évolution des besoins quantitatifs et qualitatifs du lapin est présentée, en mettant l'accent sur certaines particularités de l'espèce, à savoir son aptitude à mettre en valeur les fibres alimentaires et ses besoins en eau par rapport à la matière sèche ingérée. Un autre aspect tout aussi fondamental de la réussite de la cuniculture intensive est aussi longuement abordé, à savoir les principales maladies et leur prévention et traitement : pathologies respiratoires, maladies digestives, etc. Un dernier chapitre est consacré aux avancées de la sélection en cuniculture, en allant des méthodes traditionnelles de génétique quantitative jusqu'aux évolutions actuelles qui se traduisent par le développement des outils moléculaires et de leurs applications, notamment la génomique.

Rédigé dans un style scientifique abordable à une majorité de lecteurs, l'ouvrage devrait intéresser un vaste lectorat, aussi bien des acteurs de la filière de la cuniculture, que des étudiants ou des chercheurs s'investissant dans ce domaine, ainsi que les curieux, de plus en plus nombreux, attirés par cette espèce et par son élevage domestique. Très complet et abondamment illustré, l'ouvrage s'avère de lecture aisée, d'autant qu'un glossaire détaillé permet de retrouver les définitions de tous les mots techniques utilisés dans le texte. L'ouvrage aurait cependant été plus exhaustif par la présentation des nombreux bienfaits diététiques de la viande de lapin, ainsi que par l'évocation de systèmes d'élevage moins intensifs, qui pourraient intéresser des opérateurs économiques dans des pays moins évolués.

Mohamed Taher Sraïri
Institut Agronomique et Vétérinaire Hassan II, BP 6202,
1, rue Allal Al Fassi Madinate Al Irfane, 10101,
Rabat, Maroc
mt.srairi@iav.ac.ma

